\title{
Australia's game-changing fast radio burst hunter
}

\author{
The Australian Square Kilometre Array Pathfinder will be a key tool in future searches for fast radio bursts and \\ other transient phenomena, and is already reaping rewards, explains Principal Engineer Keith Bannister.
}

\section{T} he Australian Square Kilometre Array Pathfinder (ASKAP) is a 36-dish radio interferometer situated about $800 \mathrm{~km}$ north of Perth, Western Australia ${ }^{1}$. ASKAP is a survey instrument, optimized for wide-field surveys of the sky and its science objectives involve radio observations of the Milky Way, galaxies in continuum and polarization, and neutral hydrogen spectral lines in emission and absorption. ASKAP will also search for transient and variable radio sources, such as fast radio bursts (FRBs) and afterglows of the neutron-star mergers also observed by the Laser Interferometer Gravitational-Wave Observatory (LIGO).

ASKAP's killer feature is its large field of view, which is achieved through the use of relatively small (12 m) dishes, combined with an innovative phased-array feed (PAF) receiver. Each PAF comprises 188 individual receiving elements which, after digital beamforming, can generate up to 36 dualpolarization beams. The feeds are mounted at the prime focus of each 12-m antenna, yielding a roughly 30 square degree field of view per dish, some 30 times larger than possible with a traditional receiver. The PAF is sensitive to radio waves with frequencies in the range $700-1,800 \mathrm{MHz}$, although at present only a $336 \mathrm{MHz}$ spectral window can be processed at any given time.

Support for searching for FRBs was designed in at the early stages ${ }^{2,3}$. The primary data products for FRB searching are fast-dump autocorrelations (that is, power measurements) from each antenna. These correlations are produced by the digital beamformers with an integration time of $\sim 1 \mathrm{~ms}$ and frequency resolution of $1 \mathrm{MHz}$. While these specifications are relatively coarse when compared with other instruments, they result in a very manageable data volume: data can be processed in a single GPU node, significantly simplifying the required software and operations.

ASKAP supports two modes of operation for FRB searches. In the standard mode, all antennas point in the same direction. Data streams from each antenna are averaged together to produce an 'incoherent sum' that is searched for FRBs. With all 36 antennas operating, the standard mode will yield the ability to detect FRBs brighter than about 3 Jy over a $30 \mathrm{deg}^{2}$ field. It also has the

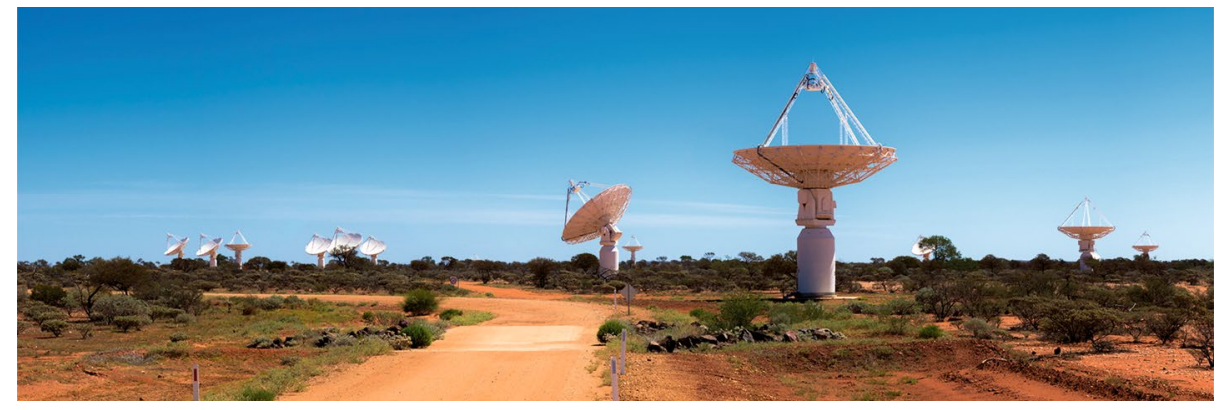

Fig. 1| ASKAP searching for FRBs in fly's-eye mode. Credit: Kim Steele

advantage of operating simultaneously with standard interferometric observations. In the alternative 'fly's-eye mode', all of the antennas are pointed in different directions (Fig. 1), and data from each antenna are searched independently. This mode yields a whopping $1,000 \mathrm{deg}^{2}$ field of view at the expense of a roughly six times poorer sensitivity.

Until now, most of the FRB searching has been done in the fly's-eye mode, with only a fraction (eight) of the full complement of ASKAP antennas. Using this configuration, ASKAP has detected 26 FRBs to date. Ryan Shannon et al. ${ }^{4}$ reported the discovery of 19 of these over an eight-month period, almost doubling the known population that had been amassed by the world's telescopes combined over the previous ten years. By comparing these with a similar number of FRBs detected by the Parkes 64-m radio telescope, Shannon et al. showed that the brighter (higher fluence) ASKAP FRBs have, on average, lower dispersion measures (DMs) than the fainter Parkes FRBs. The existence of this dispersionfluence relation suggests that the DMs of FRBs indicate their distance. Shannon et al. found no repeat bursts from any of the detected FRBs in over 12,000 hours of observations, strongly hinting that the repeating $\mathrm{FRB}^{5}$ is a very different object from one-off FRBs.

The fact that ASKAP detects the brightest FRBs is a useful property; these FRBs are on average closer, so their hosts will be easier to study. Marcin Sokolowski et al. (manuscript in preparation) recently took advantage of this property by using the Murchison Widefield Array to shadow some of ASKAP's brightest FRBs: because they were so bright in the ASKAP frequency range, Sokolowski et al. were able to set strong limits on the existence of low-frequency emission.
Another useful property of the PAFs is that most ASKAP FRBs are detected in multiple, adjacent beams. Perhaps surprisingly, this enables the position and brightness of the FRB to be determined much more accurately than is possible with the much larger Parkes radio telescope. This allowed Shannon et al. to precisely measure the fluence of the ASKAP sample, and Mahoney et al. (manuscript in preparation) to infer a possible host galaxy for one of the lowest-DM FRBs known: FRB 171020 may have come from ESO 601-G036.

In the next few months, ASKAP will be running with the full complement of 36 antennas in the standard mode to search for FRBs. This affords an advantage: when an FRB is detected, we will be able to obtain an accurate position for it by using a few seconds of data stored in circular buffers in the beamformers. In this mode we should detect an FRB every few days and localize it to within a few arcseconds, sufficient to determine the likely host galaxies for many tens of FRBs per year.

\section{Keith W. Bannister}

CSIRO Astronomy and Space Science, Epping,

New South Wales, Australia.

e-mail: keith.bannister@csiro.au

Published online: 31 October 2018 https://doi.org/10.1038/s41550-018-0597-7

\footnotetext{
References

1. Johnston, S. et al. Exp. Astron. 22, 151-273 (2008).

2. Macquart, J. et al. Publ. Astron. Soc. Australia 27, 272-282 (2010).

3. Clarke, N., D'Addario, L., Navarro, R. \& Trinh, J. J. Astron. Instrum. 3, 1450004 (2014).

4. Shannon, R. M. et al. Nature https://doi.org/10.1038/s41586-0180588-y (2018).

5. Spitler, L. G. et al. Nature 531, 202-205 (2016).
} 